\title{
NEAR-NAVIGATION GRADE QUAD MASS GYROSCOPE WITH Q-FACTOR LIMITED BY THERMO-ELASTIC DAMPING
}

\author{
S. Askari ${ }^{*}$, M.H. Asadian, K. Kakavand, and A.M. Shkel \\ MicroSystems Laboratory, University of California, Irvine, CA, USA
}

\begin{abstract}
This paper presents experimental results on the noise performance of an ultra high quality factor (Q-factor) micromachined silicon Quad Mass Gyroscope (QMG). The device was vacuum sealed at below $0.1 \mathrm{mTorr}$ in an LCC package with activated getters, demonstrating the Q-factor better than 1.7 million, along the drive and sense modes, at the $1.6 \mathrm{kHz}$ center frequency. A bias stability of $0.09 \mathrm{deg} / \mathrm{hr}$ and Angle Random Walk (ARW) of $0.015 \mathrm{deg} / \mathrm{rt}$-hr were demonstrated, without any thermal compensation or post-processing of data. To the best of our knowledge, the result presented in this paper is the highest reported Q-factor for MEMS silicon gyroscopes, now demonstrating a true potential to achieve the navigation-grade performance.
\end{abstract}

\section{INTRODUCTION}

High Q-factor, isotropy of stiffness, and symmetry of damping along the drive and sense modes are the essential attributes of high precision Coriolis Vibratory Gyroscopes (CVGs). Q-factor maximization of the drive- and sense-mode is a key to augment performance of the mode matched or nearly-matched CVGs.

In our previous work, a QMG with as-fabricated frequency mismatch of $0.2 \mathrm{~Hz}$ and Q-factor of 1.17 million has been demonstrated with $0.88 \mathrm{deg} / \mathrm{hr}$ in-run bias stability and ARW of $0.06 \mathrm{deg} / \mathrm{rt}-\mathrm{hr}$, operating in force-rebalance (FRB) mode [1]. A vacuum sealed silicon Disk Resonator Gyroscope (DRG) has been reported with a Q-factor as high as 80,000 at $14 \mathrm{kHz}$ center frequency, reporting in-run bias stability of $0.012 \mathrm{deg} / \mathrm{hr}$ and ARW of $0.002 \mathrm{deg} / \mathrm{rt}-\mathrm{hr}$ with active temperature control and compensation [2]. A Q-factor of up to 1.38 million has been measured on bulk acoustic wave $(\mathrm{m}=3)$ degenerate modes at 2.745 $\mathrm{MHz}$ center frequency, in an actively controlled vacuum chamber [3]. A Q-factor as high as 2.7 million has been demonstrated on a vacuum sealed single-axis silicon resonator at $570 \mathrm{~Hz}$ resonant frequency [4].

In this work, we present the most recent characteristics of a vacuum sealed Quad Mass Gyroscope (QMG) packaged in a ceramic Leadless Chip Carrier (LCC) with getter activation. Device was operated in an open-loop rate mode, demonstrating a Q-factor of better than 1.7 million. Figure 1 demonstrates the ringdown results, showing the Q-factor of 1.7 million and 1.8 million along the $\mathrm{X}$ - and $\mathrm{Y}$-axis, respectively. The open-loop rate mode characterization was performed in the lab conditions with phase-locked loop (PLL) and amplitude gain control (AGC) loops, without any additional calibration or compensation loops.

\section{RATE AND RATE-INTEGRATING GYROSCOPES}

In the rate operation mode, the scale factor is amplified by the quality factor of the sense mode, thus offering a higher ratesensitivity and a reduced Mechanical-Thermal Noise (MTN). The theoretical MTN angle random walk (ARW) for the gyroscope in operating in the open-loop mode is [5]:

$$
\mathrm{ARW}_{\mathrm{MTN}} \approx \sqrt{\frac{k_{B} T \omega_{y}}{A M \omega_{x}^{2} Q_{y}}\left[1+\left(\frac{Q_{y}\left(\omega_{y}^{2}-\omega_{x}^{2}\right)}{\omega_{x} \omega_{y}}\right)^{2}\right]^{-1}}\left(\frac{\mathrm{rad}}{\sqrt{\sec }}\right)
$$

Here, $\mathrm{k}_{\mathrm{B}}$ is the Boltzmann's constant, $M$ is the gyro mass, $T$ is the operating temperature measured in Kelvins, $\omega_{\mathrm{x}}$ and $\omega_{\mathrm{y}}$ are the drive and sense resonance frequencies measured in $\mathrm{rad} / \mathrm{sec}$, and $\mathrm{Q}_{\mathrm{y}}$ is the sense-mode quality factor. When the device is operated in the angular rate mode, the higher the quality factor of the sense mode and the lower the frequency split between the drive- and the sense-modes, the lower ARW and the higher signal-to-noise ratio of the gyro response.

In the rate-integrating operation mode, or the whole-angle (WA) mode, the Coriolis force transfers energy between two orthogonal degenerate vibratory modes and allows free precession of the sensing element. The resolution of the angle measurement is derived in [6], and is limited by the anisoelasticity $(\Delta \omega)$ and anisodamping $\Delta(1 / \tau)$. Thus, maximization of the energy dissipation time constant, matching modal frequencies and symmetry of the damping are the defining factors for the angular rate drift in an angle measuring gyroscope. The bias drift can be expressed as

$$
\Omega_{\text {drift }} \approx \frac{1}{2} \Delta\left(\frac{1}{\tau}\right)+\frac{1}{2} \Delta \omega
$$

Therefore, a high performance MEMS gyroscope with an interchangeable rate and rate-integrating operation mode must possess the high Q-factor degenerate modes in stiffness and damping.

\section{VACCUM SEALED QUAD MASS GYROSCOPE}

A Quad Mass Gyroscope (QMG) is a device with four symmetrically decoupled "tines" that operates in a dynamically balanced anti-phase degenerate mode [4]. The anti-phase motion of multiple masses assures minimization of the net reaction forces and moments on the anchors, which mitigates the energy loss through the substrate. Mechanical stability and mode ordering are enabled by an outer lever synchronization mechanism and inner secondary beam resonators. Four pairs of beam resonators provide the mass coupling, while widening the frequency separation between desired anti-phase mode and parasitic in-phase modes, shifting the in-phase modes to higher frequencies for common mode rejection of linear accelerations and reduction of the mode conversion losses [7].

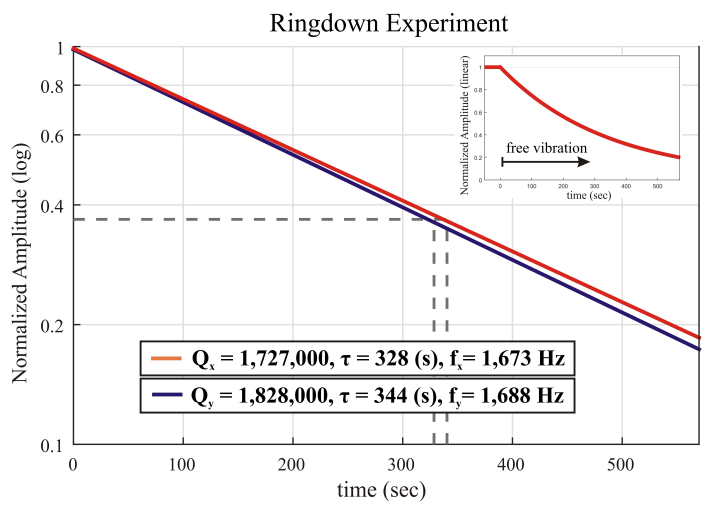

Figure 1: Experimental results of ring-down of a vacuum sealed $Q M G$ device under test. The exponential fit to the data shows the energy decay time at (1/e) normalized amplitude.

254 Solid-State Sensors, Actuators and Microsystems Workshop Hilton Head Island, South Carolina, June 5-9, 2016 

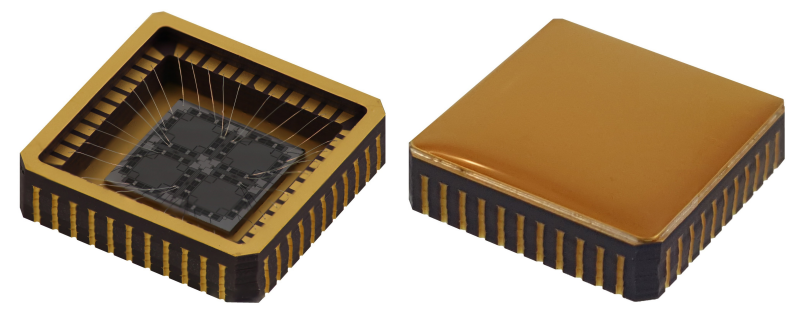

Figure 2: $8.6 \mathrm{~mm}$ footprint Quad Mass Gyroscope ( $Q M G$ ) is die attached and wire bonded. The device is vacuum sealed with an activated getter material in the LCC package.

QMGs were fabricated using Silicon-on-Insulator (SOI) process with a $100 \mu \mathrm{m}$ device layer, $5 \mu \mathrm{m}$ buried oxide layer, and a $500 \mu \mathrm{m}$ handle wafer. After fabrication and dicing, individual sensors were attached to LCC packages using eutectic bonding. Packages were dehydrogenated at $400{ }^{\circ} \mathrm{C}$ in a vacuum chamber for 3 hours prior to the die attachment. The dehydrogenation process effectively improved desorption of the trapped gas molecules in the ceramic packages. A well-suited and vacuum compatible eutectic alloy composed of $80 \%$ gold and 20\% tin (AuSn 80/20) was used for the die attachment.

QMG sensors were sealed in vacuum at $<0.1$ mTorr using an SST 3150 sealing furnace. The in-house sealing process consists of three main steps: (1) a long ( $>24$ hours) vacuum bake-out step at $220{ }^{\circ} \mathrm{C}$ to effectively eliminate moisture and adsorbed water molecules on surfaces of the package, the lid, and the device, (2) activation of the deposited thin film getter material (SAES PageLid $($ ) to maintain the high vacuum inside the sealed cavity and enable a long-term stability, and (3) thermal reflow of the eutectic AuSn $80 / 20$ solder perform at $350^{\circ} \mathrm{C} \sim 370^{\circ} \mathrm{C}$ for a defectfree bond between the Kovar lids and the ceramic packages. The Q-factors above 1 million were repeatedly achieved on QMGs using the developed sealing process, [8]. Table 1 summarizes the frequency response characteristics of four recently vacuum sealed devices, with the device \#1 being the focus of this study.

Table 1: $Q M G$ device parameter extraction after vacuum seal with activated getter, exhibiting high quality factor despite their high frequency split. (Device\# 1 studied in this paper).

\begin{tabular}{|c|c|c|c|}
\hline $\begin{array}{c}\text { Device } \\
\#\end{array}$ & $\begin{array}{c}\text { Center } \\
\text { Frequency (Hz) }\end{array}$ & $\begin{array}{l}\text { Frequency } \\
\text { Split (Hz) }\end{array}$ & Q-factor \\
\hline 1 & $\begin{array}{l}\text { X-axis: } 1,673 \\
\text { y-axis: } 1,688 \\
\end{array}$ & 15 & $\begin{array}{l}1,727,000 \\
1,828,000\end{array}$ \\
\hline 2 & $\begin{array}{l}\text { x-axis: } 2,120 \\
\text { y-axis: } 2,085\end{array}$ & 35 & $\begin{array}{l}1,206,000 \\
1,102,000 \\
\end{array}$ \\
\hline 3 & $\begin{array}{l}\text { x-axis: } 2,133 \\
\text { y-axis: } 2,089\end{array}$ & 44 & $\begin{array}{l}1,130,000 \\
1,040,000\end{array}$ \\
\hline 4 & $\begin{array}{l}\text { x-axis: } 2,142 \\
\text { y-axis: } 2,098\end{array}$ & 44 & $\begin{array}{l}1,141,000 \\
1,125,000\end{array}$ \\
\hline
\end{tabular}

\section{ENERGY DISSIPATION MECHANISMS}

Energy dissipation in microsystems, due to internal and external friction, defines the performance limit of vibratory systems. For instance, the Q-factor is a parameter of interest in resonators and CVG devices. The total Q-factor is calculated by taking all the losses into consideration. The primary energy dissipation mechanisms are viscous damping, anchor loss, and Thermo-Elastic Damping (TED). The effective quality factor is defined by the dominant energy loss mechanism. For more details on the dissipation mechanisms in a vibratory MEMS resonator. For example, our recent analysis in [4] explores the contributing factors of different dissipation mechanisms in resonators, collectively expressed as

$$
\frac{1}{Q_{\text {Total }}}=\frac{1}{Q_{\text {Viscous }}}+\frac{1}{Q_{\text {Ancho }}}+\frac{1}{Q_{T E D}}+\frac{1}{Q_{\text {Other }}}
$$

Thermal coupling is the local heating/cooling of a material, which undergoes tension and compression. This causes local irreversible heat transfer within flexures, anchored to the structure. TED-limited Q-factor was simulated based on a 2D model of the QMG device, resulting in Q-factors of $3.55 \times 10^{6}$ and $2.42 \times 10^{4}$ for anti-phase and in-phase modes, respectively. As demonstrated in Figure 3, due to lower spring stiffness and frequency $(1,703 \mathrm{~Hz})$ in the anti-phase mode, the stress within this vibratory mode is much lower compared to the in-phase mode. This is due to a higher stiffness, and higher frequency $(4,048 \mathrm{~Hz})$ in-phase vibratory mode, resulting in higher energy dissipation within the flexure.
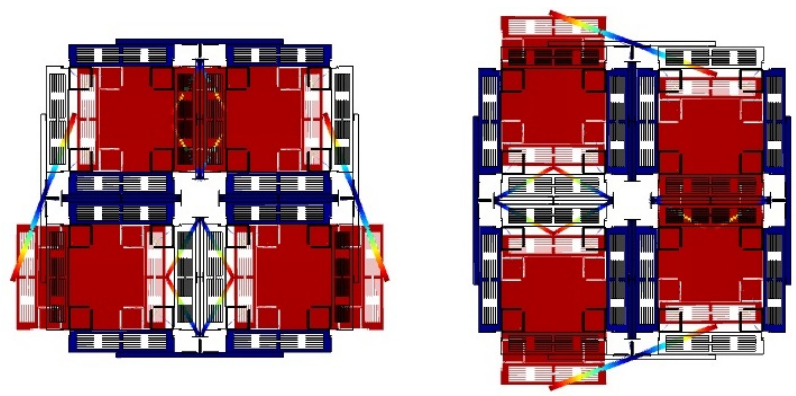

Figure 3: Finite element analysis of a Quad Mass Gyroscope $(Q M G)$, showing the fundamental TED limit of 3.54 million with a $1.7 \mathrm{kHz}$ center frequency in the anti-phase mode.

TED and anchor losses are the two dominant energy loss mechanisms in our device. The anchor loss is defined by the symmetry and dynamic balance of the structure.

\section{ELECTROSTATIC TUNING}

To extract parameters of the device, each mode was excited independently. In order to excite and track a high quality factor resonance frequency, a narrow bandwidth loop filter in the drive and sense loops were designed. For the vibratory system, this is the time, $\tau$, it takes for a settled drive amplitude to drop down under the free vibration to $1 / \mathrm{e}$ of the drive amplitude. In our experiment, the $\mathrm{X}$-mode Q-factor was measured at $\mathrm{Q}_{\mathrm{x}}=1,727,000$, which corresponds to $\tau_{\mathrm{x}}=328$ (s) at $\mathrm{f}_{\mathrm{x}}=1,673 \mathrm{~Hz}$, and along the $\mathrm{Y}$-mode it was measured at $\mathrm{Q}_{\mathrm{y}}=1,828,000$, which corresponds to $\tau_{\mathrm{y}}=344(\mathrm{~s})$ at $\mathrm{f}_{\mathrm{y}}=1,688 \mathrm{~Hz}$. Q-factor measurement was repeated over three months period, demonstrating a leak-free sealing, vacuum stability and successful activation of getter, Figure 4.

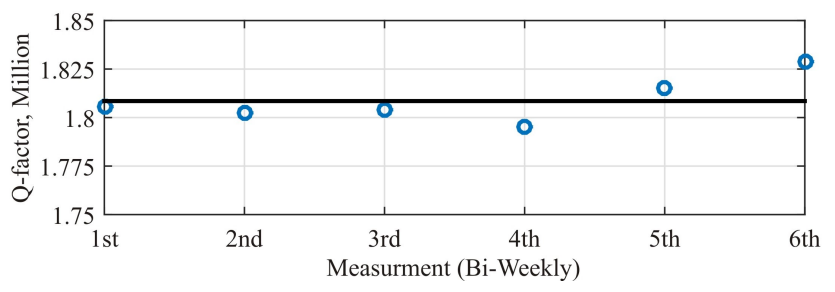

Figure 4: Stability of Q-factor was measured bi-weekly, resulting in $0.02 \mathrm{ppm}$ variations in the $Q$-factor during the period of twelve weeks. 


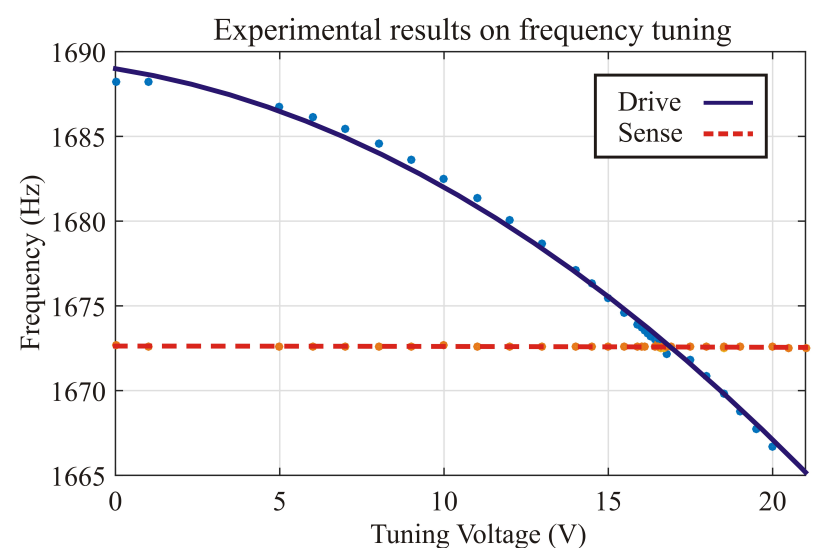

Figure 5: Experimental result of electrostatic tuning of $X-Y$ mode frequencies, by applying DC bias to the Y-mode pick-off electrodes.

Fabrication imperfections in QMGs are the primary cause of frequency mismatch between the drive and sense modes. To correct for imperfections, a frequency tuning is necessary. The QMG design consists of four proof masses with differential parallel electrodes surrounding each mass. In total, there are 16 electrodes that operate as forcer or pick-off for $\mathrm{X}$ and $\mathrm{Y}$ modes. In our experiments, a constant 10 Volt DC bias was applied to the drive forcer electrodes; the pick-off electrodes were used for DC electrostatic tuning. We also applied a DC voltage to the parallel plate electrodes of the mode with higher resonance frequency (Ymode), which decreased the resonance frequency along the $\mathrm{Y}$-axis. The center frequency of both $\mathrm{X}$ - and $\mathrm{Y}$ - modes were monitored using an off-chip lock-in amplifier. In order to monitor the frequency mismatch under tuned condition (typically $<1 \mathrm{~Hz}$ ), the following method was used. Under an open-loop condition with only the PLL locked to the drive resonance frequency with constant oscillation amplitude in the sense direction, the gyro was given an impulse disturbance. As a result of free oscillation between the principal axes of the gyro, the frequency value was detected by the sense pick-off electrodes. The frequency mismatch was extracted and minimized by monitoring the peak in power spectrum domain while actively controlling the applied DC voltage. The frequency mismatch was electrostatically tuned down to $60 \mathrm{mHz}$ using 16.58 Volts DC bias on the Y-mode electrode, with the resolution of 3.5 digits of the power supply, Figure 5. As a result of DC tuning, a drop in Q-factor from 1,828,000 to $1,521,000$ was observed along the $\mathrm{Y}$ - axis, Figure 6 .

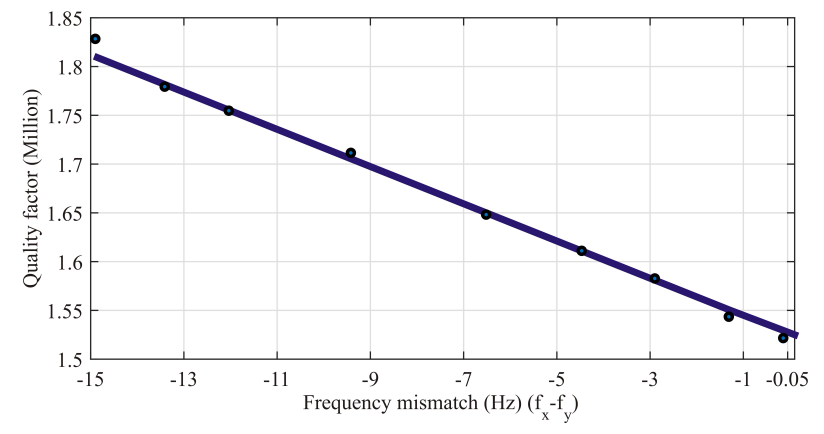

Figure 6: Experimental characterization of the Y-mode quality factor under electrostatic tuning, resulting in a drop of the $Q$ factor, while maintaining the mode-matched condition.
Figure 7 shows the increase in cross-coupling between orthogonal axes of the quad mass gyroscope (X-Y mode pick-off electrodes) as a result of electrostatic tuning. The frequency mismatch was tuned and detuned with respect to the X-mode. Due to the presence of anisoelasticity in a non-ideal gyroscope, the tuning along X-Y axes presumably resulted in an unbalanced reaction force on the anchor, leading to energy dissipation through the substrate. This is a likely explanation for changes in the orientation of the principal axes of elasticity as a result of unidirectional tuning. Therefore, a dedicated set of quadrature electrodes are likely required for the simultaneous and balanced tuning of frequency asymmetry and Quality factor.

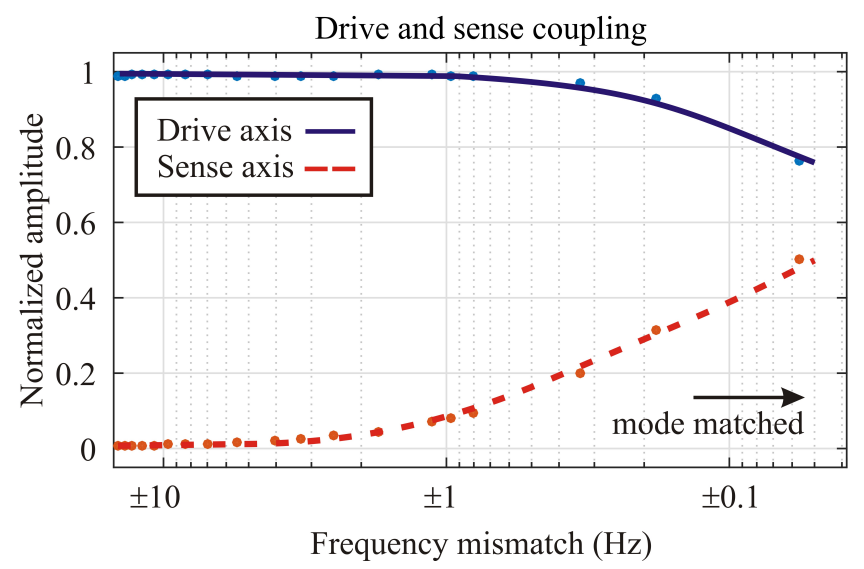

Figure 7: Drive and sense mode coupling vs. frequency mismatch, normalized to drive pick-off amplitude (Y-mode) at $15 \mathrm{~Hz}$ frequency split under a fixed actuation input.

\section{PERFORMANCE ANALYSIS}

The open loop rate mode characterization was performed with two primary loops, including Phase-Locked Loop (PLL) and Amplitude Gain Control (AGC), Figure 8. The scale factor was extracted using a rate table, with incremental step inputs of 5 $\mathrm{deg} / \mathrm{sec}$, in the clockwise and the counterclockwise direction, resulting in an open-loop scale factor of $2.1 \mathrm{mV} /(\mathrm{deg} / \mathrm{s})$, Figure 9.

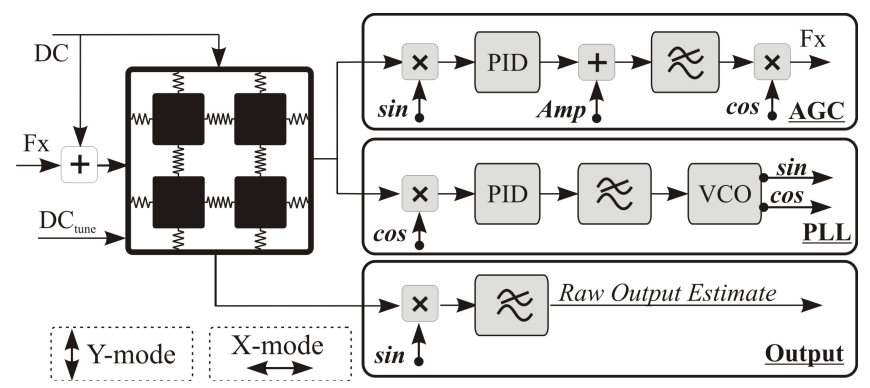

Figure 8: Overall control structure, $A G C$ and PLL were enabled along the drive axis of the device ( $X$-mode). The DC electrostatic voltage was applied to the $Y$-mode electrode.

The experiment was conducted in a lab environment without any thermal compensation. After electrostatic tuning, a bias stability of $0.09(\% / \mathrm{hr})$ and an ARW of $0.015(\% / \mathrm{h} \mathrm{hr})$ were measured, Figure 10. The result shows an improvement in signal-to-noise ratio compared to the result demonstrated previously on a $\mathrm{QMG}$ with Q-factors of 1.1 and 1.2 million and a frequency mismatch of 
$\Delta \mathrm{f}=35 \mathrm{~Hz}[8]$. As expected, the scale factor of the sensor improves as the frequency mismatch reduces and the Q-factor increases.

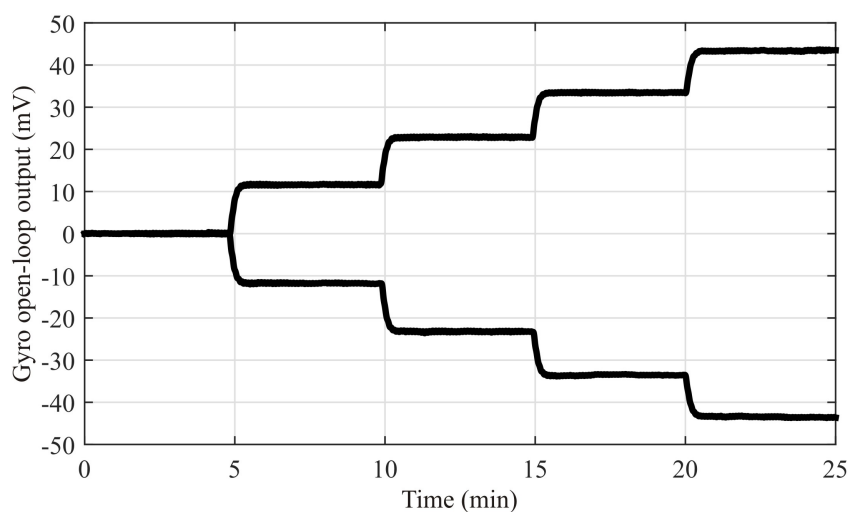

Figure 9: Rate characterization response to clockwise and counter-clockwise rotation with different step input amplitudes of $0, \pm 5, \pm 10, \pm 15, \pm 20 \mathrm{deg} / \mathrm{sec}$, revealing an open-loop Scale Factor of $2.1 \mathrm{mV} /(\mathrm{deg} / \mathrm{s})$.

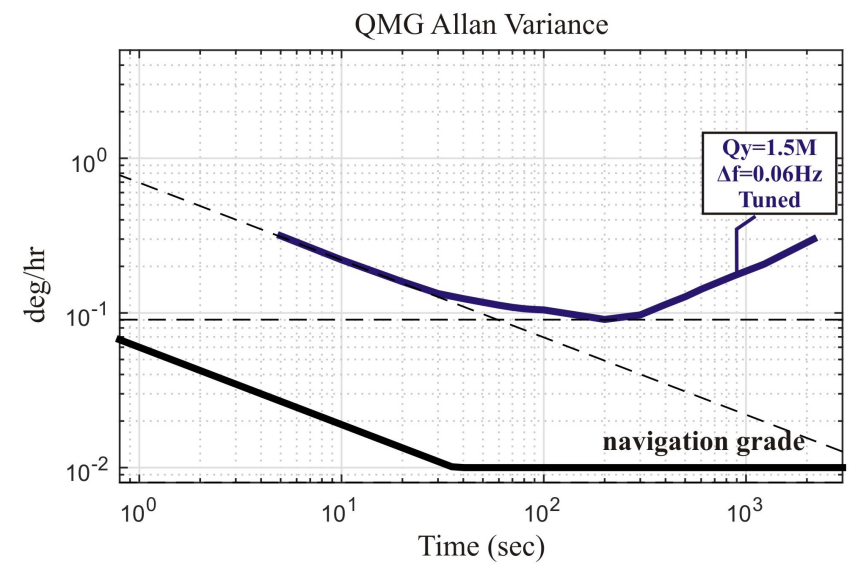

Figure 10: Performance of $Q M G$ without self-calibration algorithm ("disabled"). A vacuum sealed $Q M G$ with getter $(Q=1.5 \mathrm{M}, \Delta f=60 \mathrm{mHz}$, tuned $)$ revealed $A R W=0.015(\% / \sqrt{ } \mathrm{hr})$ and bias $=0.09(\% / h r)$ in the open-loop operation. Lower frequency mismatch and higher Quality factor are expected to reduce the inrun bias stability.

\section{CONCLUSION}

We demonstrated a vacuum sealed silicon Quad Mass Gyroscope (QMG) with the Q-factor on the level of higher than 1.7 million, with a $0.09(\% / \mathrm{hr})$ bias stability and a $0.015(\sqrt{\mathrm{hr}}) \mathrm{ARW}$ in the open-loop rate operation mode without any compensation or correction loops. The data was recorded in the lab conditions with temperature fluctuation from $23.4^{\circ} \mathrm{C}$ to $25.6^{\circ} \mathrm{C}$ with no thermal control or thermal compensation. Electrostatic tuning along the layout-defined $\mathrm{X}-\mathrm{Y}$ axes resulted in dissipation of vibration energy through the substrate, higher cross-axis coupling, and reduction of the effective Q-factor. Electrostatic tuning along the principal axis of stiffness is a necessary condition to maintain the high Q-factor while matching frequencies of the drive and sense axes. In the current design of QMG, the only electrostatic force that can be applied are along $\mathrm{X}$ - or $\mathrm{Y}$ - directions, thus an electrode with greater tuning degree of freedom is required to compensate simultaneously for frequency mismatch and alignment of the principal axes of elasticity.

\section{ACKNOWLEDGMENT}

This material is based on work supported by the Defense Advanced Research Projects Agency and U.S. Navy under Contract No. N66001-12-C-4035. Design, fabrication, and characterization were performed in UCI Microsystems Laboratory. Devices were fabricated at UCI INRF facility. Travel support has been generously provided by the Transducer Research Foundation.

\section{REFERENCES}

[1] Igor P. Prikhodko, Sergei A. Zotov, Alexander A. Trusov and Andrei M. Shkel, "Sub-degree-per-hour silicon MEMS rate sensor with 1 million Q-Factor," The 16th International Conference on Solid-State Sensors, Actuators and Microsystems (Transducers 2011), Beijing, China, 2011.

[2] Anthony D. Challoner, Howard H. Ge and John Y. Liu, "Boeing Disc Resonator Gyroscope," IEEE/ION Position Location and Navigation Symposium (PLANS 2014), Monterey, CA, 2014.

[3] Ramin Mirjalili, Hong Wen, Diego E. Serrano and Farrokh Ayazi, "Substrate-decoupled silicon disk resonators having degenerate gyroscopic modes with Q in excess of 1-million," The 18th International Conference on Solid-State Sensors, Actuators and Microsystems (Transducers 2015), Anchorage, AK, 2015.

[4] Sergei A. Zotov, Brenton R. Simon, Gunjana Sharma, Jie Han, Igor P. Prikhodko, Alexander A. Trusov and Andrei M. Shkel, "Investigation of Energy Dissipation in Low Frequency Vibratory MEMS Demonstrating a Resonator with 25 Minutes Time Constant," Solid-State Sensors, Actuators, and Microsystems Workshop (Hilton Head 2014), Hilton Head Island, SC, USA, June 8-12, 2014.

[5] Robert P. Leland, "Mechanical-thermal noise in MEMS gyroscopes," IEEE Sensors Journal, vol. 5, no. 3, pp. 493-500, 2005.

[6] David D. Lynch "Vibratory Gyro Analysis by the Method of Averaging," Proc. 2nd St. Petersburg Conf. on Gyroscopic Technology and Navigation, St. Petersburg, Russia, pp. 2634, 1995.

[7] Brenton R. Simon, Sambuddha Khan, Alexander A. Trusov and Andrei M. Shkel, "Mode ordering in tuning fork structures with negative structural coupling for mitigation of common-mode g-sensitivity," IEEE SENSORS, Busan, Korea, pp. 626-629, 2015.

[8] Sina Askari, Mohammad H. Asadian, Kasra Kakavand and Andrei M. Shkel, "Vacuum sealed and getter activated MEMS Quad Mass Gyroscope demonstrating better than 1.2 million quality factor," IEEE International Symposium on Inertial Sensors and Systems (INERTIAL), Laguna Beach, CA, USA, pp. 142-143, 2016.

\section{CONTACT}

*S. Askari, email: askaris@uci.edu 